\section{Dritter Weg möglich?}

\section{Die Forschung zum Umweltmanagement hat bisher wenig Anschluß an die öko- nomische Theorie der Unternehmung gesucht. Teilweise in Abgrenzung, teilwei- se als Ergänzung zum neoklassischen Konzept der Unternehmung sowie zum Ansatz einer "Integrativen Wirtschafts- und Unternehmensethik" scheint hier ein dritfer Weg möglich, der auf Erkenntnissen der neveren Entscheidungs- theorie beruht. Die Rekonstruktion des Normativen in faktisch stattfindenden Entscheidungsprozessen liefert wichtige Ansatzpunkte für eine sozial-ökologi- sche Unternehmenspolitik.}

$\mathrm{D}$

Von Thomas Beschorner ie dominierende Theorie der Unternehmung fußt auf der mikroökonomischen Entscheidungstheorie neoklassischer Prägung, die auf der Grundlage einer, ,as if "-Hypothese den Akteur als vollständig rational handelnd, d.h. bei substantiell unveränderlichen Präferenzen als nutzenmaximierend definiert. Bezogen auf eine sozial-ökologische Unternehmenspolitik reduziert sich betrieblicher Umweltschutz nach diesem Modell auf ,Aktivitäten, die zugleich einzelwirtschaftich vernünftig und ökologisch geboten sind“ (1). Es wird ein äußerst verkürztes Bild von Unternehmen im Sinne von Gewinnmaximierungsmaschinen gezeichnet.

Als Antithese gilt insbesondere Peter Ulrichs Konzept einer „Integrativen Wirtschafts- und Unternehmensethik“ (2). Durch eine „Transformation der ökonomischen Vernunft" versucht Ulrich der Herauslösung der ökonomischen Rationalität aus der praktischen Vernunft zu begegnen und überführt die - der Ökonomie zugrunde liegende utilitaristische in eine diskursive Etbik. Zur Realisierung schlägt Ulrich das Stakeholdermodell im Sinne einer dialogischen, konsensorientierten Unternehmenspolitik vor. Damit scheint er jedoch den ökonomischen Akteur eher diskursiv zu überfordern, als konstruktive Handlungsmöglichkeiten aufzuzeigen. Kritisch zu hinterfragen ist

\section{Beilagenhinweis}

Dieser Ausgabe liegen Informationen des Deutschen Wirtschaftdienstes und des ökom Verloges sowie der Verbände B.A.U.M. e.V. und UNTERNEHMENS GRÜN bei. Wir bitten unsere Leserinnen und Leser um freundliche Beachtung. beim Anspruchsgruppenmodell insbesondere das methodische Konstrukt der "black box", bei der lediglich die Verhaltensergebnisse betrachtet werden, nicht jedoch der Prozeß, der dieses Verhalten hervorbringt.

Im folgenden wird die Rekonstruktion des Normativen in faktisch stattfindenden Entscheidungsprozessen als ein dritter Weg vorgeschlagen. Dies bedeutet nichts anderes, als das Prinzip des „Sustainable Development" mit seinen offengelegten normativen Prämissen intraund intergenerationeller Fairneß in ohnehin schon normativ aufgeladene Entscheidungsprozesse zu implementieren. Einen möglichen theoretischen Zugang bieten neuere entscheidungstheoretische Ansätze.

\section{Komplexität und neve entschei- dungstheoretische Ansütze}

Die ökonomische Entscheidungstheorie wurde in den letzten Jahrzehnten von der Vorstellung des vollständig rational handelnden Akteurs dominiert. Als vorläufiger Höhepunkt kann dabei das Werk Gary S. Beckers gelten, der auf Grundlage des homo-oeconomicus-Modells eine allgemeine Theorie zur Erklärung menschlichen Verhaltens entwickelte. Neben empirischen Einwänden (3) formulieren neuere Entwicklungen aus der ökonomischen Systemtheorie, der Kognitionspsychologie sowie aus dem Bereich der Evolutorischen Ökonomik allerdings inzwischen ernstzunehmende theoretische Kritik an diesem Modell. Das zentrale Argument liegt in der Unmöglichkeit einer vollständigen Handlungsrationalität des/der EntscheiderIn. Wie Beckenbach in der letzten Ausgabe von Ökologisches Wirtschaften bereits verdeutlichte, gibt es gute Gründe anzunehmen, daß es sich bei dem Verhaltensmodell der neoklassischen Theorie lediglich um einen Sonderfall grundsätzlich komplexer Entscheidungen unter Unsicherbeit handelt (4). Im Gegensatz zu früheren Überlegungen des bounded-rationality-Konzeptes spielen dabei soziologische Dimensionen (Regelorientierung, Normen etc.) eine immer größere Rolle. Damit scheint eine längst überfällige Aufhebung des Gegensatzes von individualistischen und holistischen Konzeptionen möglich. Auf der Grundlage einer Prozeßbetrachtung spielen zum einen die (beschränkte) Kompetenz des Akteurs und zum anderen die Wahrnehmung der komplexen Umwelt einen zentralen Part (5). Zum einen kann der Akteur nicht alle entscheidungsrelevanten Informationen der komplexen Umwelt beschaffen; es werden vielmehr die für wichtig erachteten Daten selektiert (framing). Zum anderen wäre zu prüfen, ob die vorhandenen Daten überhaupt vollständig ,kalkuliert" werden können. Nach der Auffassung von Ronald Heiner ist gerade Unsicherheit, als Differenz zwischen Handlungskompetenz und Entscheidungsproblem (C-D-gap), die Ursache für Regelbefolgungen. Regeln, Routinen oder Normenorientierung sind somit nicht das Ergebnis eines bewußten Entscheidungskalkïls, sondern das Resultat mangelnder Flexibilität des Handlungsakteurs. Damit werden Handlungen ausgeschlossen, die unter der Situation der Sicherheit die Auszahlung erhöht hätten (6). Entscheidungen sind demnach nicht werffrei, wie die neoklassische Theorie suggeriert: „Die Werte, die man gewöhnlich meint, von den Entscheidungen getrennt halten zu können, sind konstitutive Bestandteile von Entscheidungen" (7). In seiner radikalsten Variante geht dieser Ansatz soweit, daß behauptet wird, nicht Gewohnheiten seien das Ergebnis von (unbewußten) Entscheidungen, sondern umgedreht, Entscheidungen lägen bestimmte Gewohnheiten zugrunde (8).

Eine notwendige Ergänzung wäre die Frage nach dem Zustandekommen bzw. nach der Veränderung von Gewohnheiten. Einerseits scheinen bestimmte Weltbilder, Religion o.ä. Regeln „vorgeordnet“, andererseits kann angenommen werden, daß sich das Verhalten eines Akteurs nicht im Vakuum, sondern in Abhängigkeit zum Verhalten anderer vollzieht (9). Insofern scheinen sowohl Regeln als auch Entscheidungen Sozialität zu implizieren. Der Übergang von einer Verhaltensweise zu einer anderen, somit innovatives Verbalten findet durch "trial and error", durch Imitation und durch Rekombination bereits bekannter Aspekte statt (10). Allen drei Methoden ist 
jedoch die Selektion aufgrund bekannter Interpretationsmuster gemein. Dieser Sachverhalt weist auf eine ausgesprochene Pfadabhängigkeit von Entwicklungsprozessen hin.

Überträgt man die Erkenntnisse der neueren Entscheidungstheorie auf betriebliche Prozesse, so ergeben sich für eine sozial-ökologische Unternehmenspolitik zwei zentrale Ansatzpunkte zur Implementierung des Nachhaltigkeitsprinzips. Zum einen lassen sich Handlungsempfehlungen zur Ausgestaltung des sozialen Systems entwickeln, zum anderen ist eine Erweiterung des Stakeholderkonzepts möglich.

\section{- Rekonstruktion des Normativen als sozial-ökologische Unterneh- menspolitik}

Soziale Prozesse im allgemeinen und betriebliche Entscheidungsprozesse im besonderen werden nicht nur zweckrational „,kalkuliert", sondern vorrangig traditional im Sinne von Gewohnheiten und Regeln "gelebt". Insofern sind Werte und Normen in Unternehmen immer und überall vorhanden und haben insbesondere im Rahmen des strategischen und normativen Managements eine handlungsleitende Funktion inne. Daß diese These durchaus empirischen Faktizitäten standhalten kann, zeigen nicht nur Untersuchungen zur Entscheidungsorientierung von Führungskräften (11), sondern auch neuere Erhebungen aus dem Bereich der Unternehmenskultur (12). Werte und Normen finden sich in Unternehmen ganz konkret als Unternehmensphilosophien und -kulturen, als Leitsätze, Rituale, in unzähligen Regeln, aber auch in nach außen getragenen Normenbezügen, z.B. ISO 9000 oder der Umwelterklärung nach dem EGÖko-Audit-System. Die Entwicklungs- und insofern auch die Überlebensfähigkeit von Unternehmungen wird im hohen Maße von deren Reflexionsfähigkeit über Gewohnheiten und innerbetriebliche Normen abhängig sein. Die ökologische Entwicklungsfähigkeit dürfte vom innerbetrieblichen sozialen Bezugsrahmen (Sozialorientierung) abhängen (13). Könnte die positive Korrelation von Sozial- und Umweltorientierung auch in konventionellen Unternehmungen nachgewiesen werden, so ergäben sich - vor dem Hintergrund pfadabhängiger Entwicklungsprozesse - konkrete Handlungsspielräume. Es kann angenommen werden, daß auch Unternehmen einem Prozeß ,organisationaler Sozialisation" unterliegen und sich innovatives Verhalten auf der Grundlage bekannter Interpre- tationsmuster vollzieht. Es ist deshalb eingehender zu prüfen, inwieweit eine Sozialorientierung in Unternehmen als wichtiger Nährboden zur Entwicklung einer sozial-ökologischen Unternehmenspolitik beiträgt und welche Gestalt ein angemessener sozial-ökologischer Entwicklungspfad haben sollte.

Neben einer veränderten Perspektive hinsichtlich der Beurteilung der internen Entscheidungsprozesse ergibt sich - wie bereits angedeutet - auch eine andere Heuristik für die Wahrnehmung der Unternehmensumwelt. Neben der üblichen Betrachtung von Unternehmen im Beziehungsgeflecht unterschiedlicher Stakeholder veranschaulicht die Analyse, daß Unternehmen nicht nur in ein Interdependenz-, sondern darïber hinaus auch in ein Wertegefïge eingebunden sind (14). Schließlich, so kann vermutet werden, gewährleistet nicht nur eine adäquate Gestaltung der Binnenbeziehungen die Wahrnehmungsfähigkeit sozial-ökologischer Dimensionen, vielmehr kann auch ein ökologischer Impuls von der Unternehmensumwelt einen notwendigen Veränderungsprozeß anstoßen. Insofern ist ein interdependenter Zusammenhang zwischen Sozial- und Umweltorientierung zu vermuten.

Rudi Kurz sieht in der Beteiligung am gesellschaftlichen Suchprozeß nach einer nachhaltigen Wirtschaftsweise nicht nur eine sittliche Dimension, sondern vor allem langfristige strategische Vorteile für Unternehmen. Die Existenzsicherung von Unternehmen wird dabei insbesondere durch eine verbesserte Wahrnehmungsfähigkeit der Unternehmensumwelt, durch eine erhöhte Anpassungsfähigkeit an veränderte Rahmenbedingungen und durch eine gesteigerte Gestaltungsfähigkeit der Umweltbedingungen ermöglicht (15). Gleichwohl ermöglicht der hier vorgestellte Zugang - mit den Worten Max Webers - auch die Reflexion traditionalen Handelns im Sinne einer Wert- versus Zweckrationalität, womit Unternehmensethik auch jenseits ökonomischer Klugheitserwägungen möglich wird.

\section{Anmerkungen}

(1) Freimann, Jürgen: Betriebliche Umweltpolitik. Bern, Stuttgart, Wien 1996, S. 359-360.

(2) Vgl. insbesondere Ulrich, Peter: Integrative Wirtschaftsethik. Grundlagen einer lebensdienlichen Ökonomie. Bern, Stuttgart, Wien 1997.

(3) Vgl. dazu die Übersicht bei Conlisk, John: Why Bounded Rationality? In: Journal of Economic Literature Vol. 34 (1996), S. 669-700.

(4) Beckenbach, Frank: Paradigmatische Neuorientierung.
Ökologisch-ökonomische Komplexitütsforschung im Überblick. In: Ökologisches Wirtschaften 3-4/98, Spezial, S. 1-3.

(5) Tamborini, Roberto: Knowledge and Economic Behoviour. A Constructivist Approach. In: Journal of Evolutionary Economics, Vol. 7 (1997), No. 1, S. 49-72.

(6) Heiner, Ronald A.: The Origin of Predictable Behavior. In: American Economic Review, Vol. 73 (1983), №. 4, S. 560-595.

(7) Priddat, Birger: Statt einer Einleitung: Essay über Unternehmensphilosophie - und darüber, was sie nicht ist. In: Lohmann; Schmidt 1996 (Anm. 12), S. 12.

(8) Hodgson, Geoffrey M.: The Approach of Institutional Economics. In: Journal of Economic Literature, Vol. 36 (1998), S. 166-192.

(9) Witt, Ulrich: Überlegungen zum gegenwärtigen Stand der evolutorischen Ökonomik. In: Bernd Biervert und Martin Held (Hrsg.): Evolutorische Ökonomik. Tübingen 1992, S.23-55.

(10) Popper, Karl R.; John C. Eccles: Das Ich und sein Gehirn, München 1989.

(11) Ulrich, Peter; Ulrich Thielemann: Ethik und Erfolg, Bern, Stuttgart, Wien 1991 sowie Heine, Hartwig, Rüdiger Mautz: Öffnung der Wogenburg? - Antworten von Chemiemanagern auf ökologische Kritik, Berlin 1995.

(12) Lohmann, Karl R.; Thomas Schmidt (Hrsg.): Werte und Entscheidungen im Management, Marburg 1996 sowie Flieger, Burghard: Partizipative Umweltunternehmen als Lernfelder für die Politik und Wirtschaft von morgen. In: Ökologie und Partizipation, Bonn 1997, S. 219-245.

(13) Gellrich, Carsten; Alexandra Luig; Reinhard Pfriem: Ökologische Unternehmenspolitik: von der Implementierung zur Fähigkeitsentwicklung. In: Martin Birke et al. (Hrsg.): Handbuch Umweltschutz und Organisation. München, Wien 1997, S. 523-562.

(14) Beschorner, Thomas: Informationssysteme und Theorie der Unternehmenspolitik - zum Beziehungsgefüge von Erklärungs- und Gestaltungsansätzen einer sozial-ökologischen Betriebswirtschaftslehre, Werkstattreihe Betriebliche Umweltpolitik, Band 11, Kassel 1998.

(15) Kurz, Rudi: Unternehmen und nachhaltige Entwicklung. In: Jahrbuch Ökonomie und Gesellschaft, Nachhaltigkeit in der ökonomischen Theorie, Frankfurt 1997, S. $78-125$.

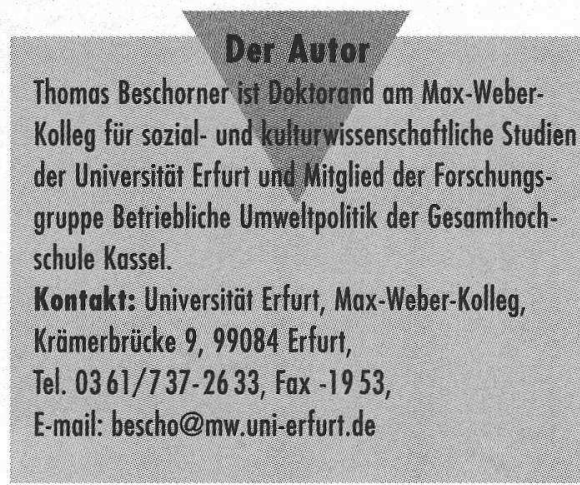


(c) 20I0 Authors; licensee IÖW and oekom verlag. This is an article distributed under the terms of the Creative Commons Attribution Non-Commercial No Derivates License (http://creativecommons.org/licenses/by-nc-nd/3.o/), which permits unrestricted use, distribution, and reproduction in any medium, provided the original work is properly cited. 